\title{
Second virial coefficient for the exponent-spline-Morse-spline-van der Waals potential and its application
}

\author{
E. Somuncu ${ }^{1}$ and B.A. Mamedov ${ }^{2}$ \\ ${ }^{1}$ Affiliation not available \\ ${ }^{2}$ Gaziosmanpasa University
}

June 22, 2021

\begin{abstract}
An analytical expression for the second virial coefficient based on an exponent-spline-Morse-spline-van der Waals (ESMSV) potential is presented here for use in defining the thermodynamic properties of rare gases. Our method is established based on a series expansion of the exponential function, Meijer function, gamma function, binomial function, and hypergeometric function. Numerical approaches have commonly been used for the evaluation of the second virial coefficient with the ESMSV potential in the literature. The general formula obtained here can be applied to estimate the thermal properties of rare gases. Our results for the second virial coefficient based on the ESMSV potential of He-He, He-Ne, He-Ar, and He-Xe rare gases are compared with numerical calculations and experimental data, and it is shown that our analytical expression can be successfully used for other gases.
\end{abstract}

\section{Hosted file}

Second virial coeffcient ESMSV sonnn.docx available at https://authorea.com/users/350054/ articles/527314-second-virial-coefficient-for-the-exponent-spline-morse-spline-van-derwaals-potential-and-its-application 